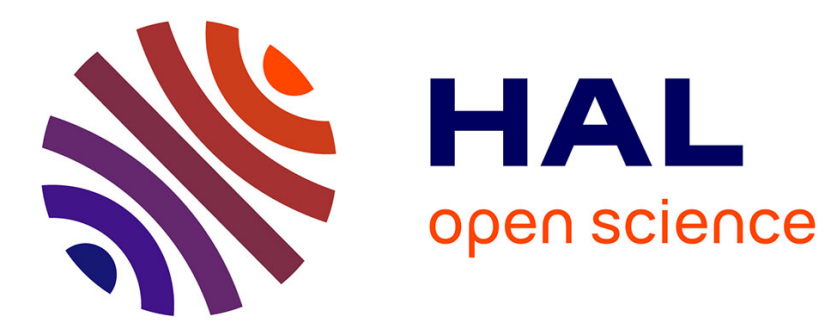

\title{
New insights into self-heating in double-gate transistors by solving Boltzmann transport equations
}

\author{
T. Thu Trang Nghiêm, Jérôme Saint-Martin, P. Dollfus
}

\section{To cite this version:}

T. Thu Trang Nghiêm, Jérôme Saint-Martin, P. Dollfus. New insights into self-heating in double-gate transistors by solving Boltzmann transport equations. Journal of Applied Physics, 2014, 116 (7), 10.1063/1.4893646 . hal-01906713

\section{HAL Id: hal-01906713 \\ https://hal.science/hal-01906713}

Submitted on 17 Jul 2019

HAL is a multi-disciplinary open access archive for the deposit and dissemination of scientific research documents, whether they are published or not. The documents may come from teaching and research institutions in France or abroad, or from public or private research centers.
L'archive ouverte pluridisciplinaire HAL, est destinée au dépôt et à la diffusion de documents scientifiques de niveau recherche, publiés ou non, émanant des établissements d'enseignement et de recherche français ou étrangers, des laboratoires publics ou privés. 


\section{New insights into self-heating in double-gate transistors by solving Boltzmann transport equations}

T. Thu Trang Nghiêm, J. Saint-Martin, and P. Dollfus

Citation: Journal of Applied Physics 116, 074514 (2014); doi: 10.1063/1.4893646

View online: https://doi.org/10.1063/1.4893646

View Table of Contents: http://aip.scitation.org/toc/jap/116/7

Published by the American Institute of Physics

\section{Articles you may be interested in}

Nanoscale thermal transport. II. 2003-2012

Applied Physics Reviews 1, 011305 (2014); 10.1063/1.4832615

Increase of self-heating effects in nanodevices induced by surface roughness: A full-quantum study Journal of Applied Physics 117, 084313 (2015); 10.1063/1.4913511

Monte Carlo simulation of Joule heating in bulk and strained silicon

Applied Physics Letters 86, 082101 (2005); 10.1063/1.1870106

Heat generation in semiconductor devices

Journal of Applied Physics 75, 942 (1994); 10.1063/1.356450

Thermal conductivity of individual silicon nanowires

Applied Physics Letters 83, 2934 (2003); 10.1063/1.1616981

Concurrent thermal and electrical modeling of sub-micrometer silicon devices

Journal of Applied Physics 79, 7353 (1996); 10.1063/1.361424

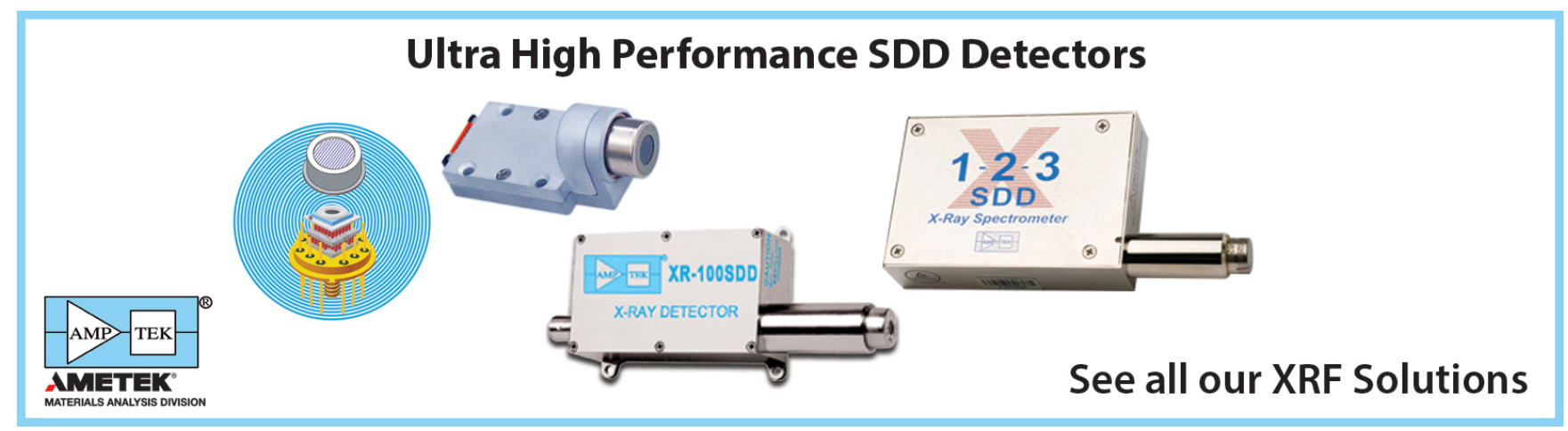




\title{
New insights into self-heating in double-gate transistors by solving Boltzmann transport equations
}

\author{
T. Thu Trang Nghiêm, ${ }^{1,2, a)}$ J. Saint-Martin, ${ }^{1}$ and P. Dollfus ${ }^{1}$ \\ ${ }^{1}$ Institute of Fundamental Electronics, UMR 8622, CNRS-University of Paris-Sud, Orsay, France \\ ${ }^{2}$ The Center for Thermal Sciences of Lyon, UMR 5008, CNRS-INSA-University of Lyon 1, \\ Villeurbanne, France
}

(Received 12 March 2014; accepted 3 August 2014; published online 20 August 2014)

\begin{abstract}
Electro-thermal effects become one of the most critical issues for continuing the downscaling of electron devices. To study this problem, a new efficient self-consistent electron-phonon transport model has been developed. Our model of phonon Boltzmann transport equation (pBTE) includes the decay of optical phonons into acoustic modes and a generation term given by electron-Monte Carlo simulation. The solution of pBTE uses an analytic phonon dispersion and the relaxation time approximation for acoustic and optical phonons. This coupled simulation is applied to investigate the self-heating effects in a $20 \mathrm{~nm}$-long double gate MOSFET. The temperature profile per mode and the comparison between Fourier temperature and the effective temperature are discussed. Some significant differences occur mainly in the hot spot region. It is shown that under the influence of self-heating effects, the potential profile is modified and both the drain current and the electron ballisticity are reduced because of enhanced electron-phonon scattering rates. (C) 2014 AIP Publishing LLC. [http://dx.doi.org/10.1063/1.4893646]
\end{abstract}

\section{INTRODUCTION}

At short gate length, Complementary Metal-OxideSemiconductor (CMOS) devices and circuits face thermal management challenges resulting from local heating due to phonon emission by hot carriers in active regions of reduced size. This phenomenon, called self-heating effect, is identified as one of the most critical issues for continuing the increase in the packing density of integrated circuits. This is especially crucial in SOI technology (silicon on insulator), where the presence of the buried insulator hinders the dissipation of heat.

At the nanoscale, the theoretical study of heating phenomena cannot be led rigorously using the macroscopic Fourier heat equation. It requires a detailed description of microscopic heat transfers in far-from local equilibrium conditions. Advanced models able to describe out-of-equilibrium transport for both electron and phonon populations together with their interactions can improve our understanding of the heating effects in nanodevices and help to optimize the thermal management.

Several previous works have used hydrodynamic models ${ }^{1}$ or TCAD simulation tools $\mathrm{s}^{2,3}$ for electrons and phonons to describe heat generation and transport in submicron silicon devices. Even if these methods provide useful and time-saving information on electro-thermal effects, the particle ensemble Monte Carlo method (MC) is the most accurate to investigate out-of-equilibrium transport regimes that occur in the hot spot region of nano-transistors, ${ }^{4}$ at least in the semi-classical limit.

From 2004, the Goodson group has developed the electron Monte Carlo (eMC) model $^{5,6}$ for heat generation and

\footnotetext{
a) Author to whom correspondence should be addressed. Electronic mail: thutrang.nghiem@gmail.com.
}

the split-flux $\operatorname{model}^{7}$ for phonon transport. Their eMC model includes the isotropic and quadratic phonon dispersion proposed in Ref. 5. Then, they coupled these two models to perform a self-consistent simulation of non-equilibrium transport in Si-FETs. ${ }^{8}$ Their coupled simulation begins with an isothermal eMC simulation at $300 \mathrm{~K}$. Then the net phonon generation rates are collected as a function of position and phonon frequency $^{5,9}$ and used as an input for the split-flux phonon transport model. The solution gives an updated distribution of phonons at each spatial position. The simulation is iterated until satisfactory convergence is reached. The model was used to study a 1D 20 nm-long-n-region in $\mathrm{a} \mathrm{n}^{+}-n-n^{+}$Si diode. The authors emphasized the role of g-LO ( $g$ type-i.e., with an exchanged wave vector of $k \approx 0.3 k_{\max }$-electron/longitudinal optical scattering mechanism) and the phonon bottleneck effect.

In 2006, Zebarjadi et al. ${ }^{10}$ coupled the Fourier heat equation and eMC simulation to study the size effect on thermoelectric properties of III-V heterostrucutre. In the work of Sadi and Kelsall, the 2D heat diffusion equation was selfconsistently coupled with a $2 \mathrm{D}$ electron eMC simulation to study the electro-thermal phenomena in SOI-Field Effect Transistors (FETs) and in Silicon-Germanium-on-Insulator metal-oxide FETs. ${ }^{11}$ They highlighted the electron velocity and the drain current degradations in a $0.1 \mu \mathrm{m}$-long-channel transistor. This approach has been extended to the case of InGaAs high-electron mobility transistors (HEMTs). ${ }^{12}$ In these $2 \mathrm{D}$ simulations, although the thermal conductivity is position dependent, the model is, by nature, unable to capture out of equilibrium thermal phenomena.

Extending the model of Lai and Majumdar for the electro-thermal effects, ${ }^{13}$ the group of Raleva et al. ${ }^{14-16}$ used a system of energy balance equations of thermal transport where the acoustic and optical phonon populations are 
distinguished. Thus, this thermal model coupled with an eMC simulation can account for the phonon bottleneck, i.e., the accumulation of optical phonons with an ultra-small group velocity in the active region of ultra-short device. This leads to a strong heating-induced drain current degradation which depends on the thermal conductivity of thin Si films that was introduced analytically in the model. ${ }^{15}$ Hatakeyama and Fushinobu ${ }^{17}$ employed a similar model to study the thermal cross-talk between the nMOS and pMOS FETs of $90 \mathrm{~nm}$ channel length that were set side-by-side to design a CMOS gate. Although these approaches-strongly efficient in terms of computational resources-capture some aspects of the out equilibrium energy transfers that are involved in sub-micron devices, they are not suitable to provide a deep microscopic insight of phonon transport ${ }^{18}$ as it is hidden by the averaged notion of effective temperature.

From our knowledge, only Kamakura et al. developed, in 2010, a MC method to solve the transient BTE for both electrons and phonons. ${ }^{19}$ They simulated a simple 1D n-i-n Si device. However, the contribution of optical phonons was neglected. The energy stored in the optical modes could be dissipated only through the conversion into acoustic modes with a relaxation time of $10 \mathrm{ps}$. The acoustic phonons were approximated by the Debye approximation with a constant velocity of $v_{a c}=5.9 \mathrm{~km} / \mathrm{s}$. The solution of transient phonon Boltzmann transport equation (pBTE) was validated by calculating the thermal conductivity obtained for various temperatures. This accurate but highly resource consuming approach has not been used to study a realistic transistor.

More recently, in 2012, by using an anisotropic relaxation time in a pBTE solver, Ni et al. ${ }^{18}$ estimated the hotspot temperature in a MOSFET. The phonon generation spectrum obtained via eMC simulation was incorporated into the anisotropic relaxation time pBTE model of Ref. 20. The phonon dispersion included six branches ( 2 transverse acoustic: TA, 1 longitudinal acoustic: LA, 2 transverse optical: TO, and 1 longitudinal optical: LO). A fully anisotropic Brillouin zone was also taken into account. However, the computational cost of this model is expensive. ${ }^{20}$

We have developed a new efficient tool that solves self-consistently the BTE for both electrons and phonons. An ensemble Monte Carlo solver for electrons is coupled with a deterministic solver of the pBTE. Our goal is to account for the role of both hot electrons and hot phonons in the selfheating effect beyond the Bose-Einstein statistics. In this work, our code is used to investigate a $20 \mathrm{~nm}$-long double gate MOSFET (DG-MOSFET). In Sec. II, the computation of the phonon generation is presented. Section III describes the phonon transport coupling procedure and the simulated structure. Sections IV and V show the results of phonon generation and the resulting effects on electron transport and device characteristics, respectively.

\section{MONTE CARLO MODEL FOR ELECTRON TRANSPORT AND HEAT GENERATION}

The electron transport was computed using a home-made Monte Carlo particle code self-consistently coupled to 2D Poisson's equation. It has been already used to study a wide range of transport problems. ${ }^{21-24}$ This method of solving the BTE offers an accurate description of out-of-equilibrium phenomena and allows us to extract the phonon emission and absorption spectra in both real space and energy space. The conduction band of silicon is described through an analytic non-parabolic model for the six ellipsoidal $\Delta$ valleys. These analytic bands give a density of states (DOS) close to that of a full-band description below about $1.5 \mathrm{eV}{ }^{8}$ Degeneracy effects and quantum corrections ${ }^{24}$ were not included here. All details of the band structure, the deformation potentials, and the scattering parameters used for acoustic phonon, intervalley phonon, ionized impurity, and oxide interface roughness scattering used in our simulator may be found in Ref. 25. It should be emphasized that the evolution of the mobility as a function of the doping level and the electric field computed with our simulator reproduces experimental results in unstrained (and strained) bulk-Si. ${ }^{25}$

In our MC model for electrons, the phonon mode dispersions used to efficiently compute the intervalley scattering rates are wave vector-independent, as shown in Fig. 1(a). The phonon wave vector $\vec{q}$ involved in an electron-phonon scattering event is deduced from the difference between initial and final electron wave vectors. To obtain realistic emission and absorption phonon energy spectra from this phonon wave vector, the phonon energy is updated a posteriori according to the appropriate quadratic dispersion $\omega(q)$ proposed in Ref. 5, plotted in Fig. 1(b), within an isotropic and spherical approximation of the first Brillouin zone. This procedure is applied for all acoustic and optical phonon modes. The resulting phonon energies are thus spread properly around the phonon energy implemented in the MC code.

As this approach does not intrinsically ensure energy conservation between electrons and phonons, we have checked its physical consistency in bulk Silicon material under uniform electric field $\vec{E}$. During the MC simulation, all phonon absorption and emission events are recorded. In Fig. 2, the resulting net power generation is compared to the product of the current density $\vec{J}$ and the electric field $\vec{E}$. In steady-state regime, the sum of the four "modified" phonon mode contributions corresponds exactly to the heat generation and our approach appears fully relevant. The most important modes involved in the generation process are LA and TO, with a contribution up to more than $40 \%$ each for all electric fields.

It should be mentioned that the previous work of Pop et al., ${ }^{6}$ using a different set of deformation potentials, has reported that the main phonon mode contribution comes from the LO branch. As a wide range of values have been reported in the literature for deformation potentials (see, for instance, Refs. 8 and 26), which are empirically determined to fit experimental mobility, the best set of parameters largely remains an open theoretical issue. ${ }^{27}$

\section{PHONON TRANSPORT}

To study the heat transport at the nanometer scale, this work is based on the Boltzmann formalism dedicated to phonons. $^{28} \mathrm{We}$ will focus on the steady state-regime in a one-dimensional (1D) real space. 


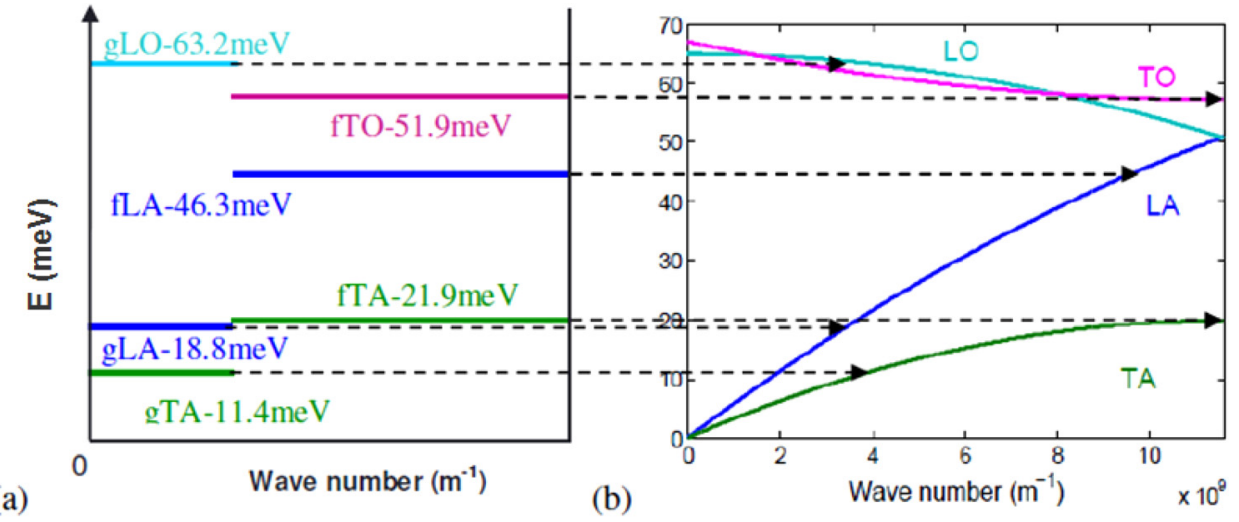

FIG. 1. (a) Intervalley phonon energies used in eMC simulation: $\mathrm{L}(\mathrm{T})-\mathrm{A}(\mathrm{O})$ stands for longitudinal (transverse) acoustic (optical) phonon and $\mathrm{g}(\mathrm{f})$ for intervalley scattering mechanism. (b) Quadratic and isotropic phonon dispersion used in this work, according to Ref. 17. Dashed lines and arrows indicate the energy and wave vector of the 6 phonon modes involved in intervalley scattering.
For each phonon mode (LA, TA, LO, or TO), the transport equation can be written as

$$
\vec{v}_{g, \mathrm{~s}}(\vec{q}) \cdot \vec{\nabla}_{\vec{r}} \cdot N_{\mathrm{s}}(\vec{r}, \vec{q})=-\left.\frac{\partial N}{\partial t}\right|_{\text {coll }}+G_{s}(\vec{r}, \vec{q}),
$$

where the index $s$ refers to the phonon mode, $\vec{q}$ is the wave vector, $\vec{v}_{g, \mathrm{~s}}(\vec{q})$ the group velocity, and $N_{\mathrm{s}}(\vec{r}, \vec{q})$ the phonon distribution. The three terms in (1) are the diffusion term (left side), the scattering term $\partial N /\left.\partial t\right|_{\text {coll }}$, and the generation term $G_{s}(\vec{r}, \vec{q})$, respectively.

\section{A. Scattering term}

To work with a tractable and linear expression for the scattering term, we used the relaxation time approximation (RTA) which leads to

$$
\frac{\partial N}{\partial t_{\text {coll }}}=-\frac{N_{\mathrm{s}}(\vec{r}, \vec{q})-N_{\mathrm{s}}^{0}\left(T_{\text {Fourier }}(\vec{r}), \vec{q}\right)}{\tau_{s}\left(T_{\text {Fourier }}(\vec{r}), \vec{q}\right)},
$$

where $N_{s}^{0}$ refers to the equilibrium distribution that should be reached in near-equilibrium transport problems. To build a

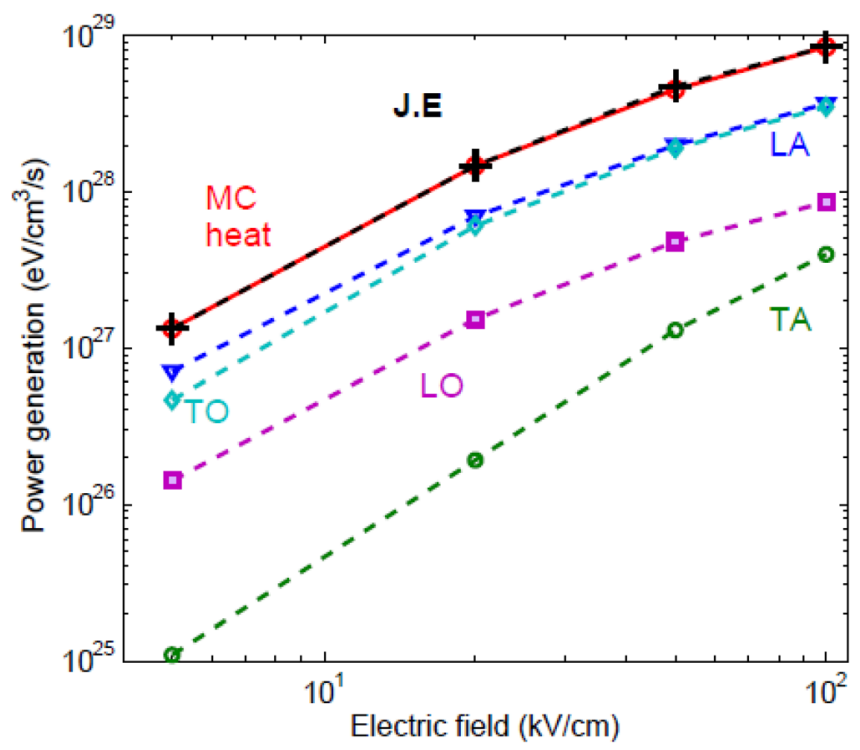

FIG. 2. Power generation per mode as a function of electric field (TA: green line and circles, LA: blue line and stars, LO: purple line and triangles, and TO: light blue line and diamonds) and comparison of total MC heat (red line and circles) with Joule effect (dashed black line and cross symbols). model relevant in both diffusive and ballistic regimes, the temperature $T_{\text {Fourier }}$ used to evaluate $N_{s}^{0}$ (according to BoseEinstein statistics) is assumed to be the same for each phonon mode and provided by the solution of the Fourier heat equation, as also considered in the pBTE solver. ${ }^{29}$

The total relaxation time $\tau_{s}\left(T_{\text {Fourier }}(\vec{r}), \vec{q}\right)$ is computed via a Mathiessen rule where the contributions of phononphonon scattering, phonon-impurity scattering and interface roughness scattering are considered. The models used to evaluate the phonon-phonon (for acoustic modes), phononimpurity and phonon-boundary (for bulk material) scattering rates are derived from the Holland model. ${ }^{30}$

The numerical values of the scattering parameters used in this work are reported in Table I. Some parameters have been tuned to reproduce experimental thermal conductivities of $\mathrm{Si}$ bulk when employing the parabolic dispersion of Ref. 9. Our model reproduces the experimental bulk silicon thermal conductivity of $149 \mathrm{Wm}^{-1} \mathrm{~K}^{-1}$ by considering phonon-phonon, phonon-boundary (for bulk), and phononimpurity scattering mechanisms (see more in Ref. 29).

In thin films of thickness $T_{S i}=20 \mathrm{~nm}$, a phonon-boundary scattering rate is added according to the model of Ref. 31 where the roughness parameter $\Delta$ has been fixed at $3 \mathrm{~nm}$. This lead to a thermal conductivity of $29 \mathrm{Wm}^{-1} \mathrm{~K}^{-1}$ in $20-\mathrm{nm}$ thick silicon films while the experimental conductivity of $24 \pm 3 \mathrm{Wm}^{-1} \mathrm{~K}^{-1}$ is mentioned in Ref. 33. It should be mentioned that this value of $\Delta$ corresponds to roughness height that is high and even unrealistic for standard $\mathrm{SiO}_{2}-\mathrm{Si}$ interface.

For the phonon-phonon scattering of longitudinal and transverse phonon (LTO) modes, a constant relaxation time of $3.5 \mathrm{ps}$ is used. This value is the average value reported in Ref. 32 in the range of $300-400 \mathrm{~K}$.

\section{B. Generation term}

Two kinds of generation processes are considered. The total generation term is thus given by

$$
G(\vec{r}, \vec{q})=G_{s}^{e-p h o n o n}(\vec{r}, \vec{q})+G_{s}^{L T O \rightarrow L T A}(\vec{r}, \vec{q}),
$$

where $G_{s}^{e-p h o n o n}(\vec{r}, \vec{q})$ is the generation term related to electron-phonon scattering. It is specific of each phonon mode. In our model, it is considered as an input to be first determined via external Monte Carlo simulation of electrons. $^{22,25}$ LTO refers to all optical phonon modes (LO and TO), and LTA refers to both LA and TA phonons. 
TABLE I. Relaxation times associated to phonon scattering mechanisms used in this work. In this table, $k_{B}$ is the Boltzmann constant, $v_{g, s}$ is the group velocity of mode $s, F$ is an average form factor, $q$ is the phonon wave vector, $\Delta$ is the roughness parameter, and $\theta_{B}$ is the incident angle of particle to the surface. The frequency $\omega_{c u t}=\omega\left(q_{\max } / 2\right)$ corresponds to the frequency at which the Umklapp scattering process of TA phonons becomes possible.

\begin{tabular}{|c|c|c|c|}
\hline \multicolumn{4}{|l|}{ Scattering type } \\
\hline Phonon-phonon & $\begin{array}{c}\text { TA-Normal (Ref. 29): } \\
\tau_{T A}^{-1}=B_{T N} \omega T^{4}, \text { with } \\
B_{T N}=10.5 \times 10^{-13} \mathrm{~K}^{-4}\end{array}$ & $\begin{array}{c}\text { TA-Umklapp (Ref. 29): } \\
\tau_{T A}^{-1}=B_{T U} \omega^{2} / \sinh \left(\hbar \omega / k_{B} T\right), \text { with } \\
B_{T U}=0\left(\text { if } \omega<\omega_{c u t}\right) \\
B_{T U}=2.89 \times 10^{-18} \mathrm{~s}\left(\text { if } \omega>\omega_{\text {cut }}\right)\end{array}$ & $\begin{array}{l}\text { LTO (Ref. 31): } \\
\tau_{L T O}=3.5 \mathrm{ps}\end{array}$ \\
\hline Phonon-impurity & & $\tau_{I}^{-1}=A \omega^{4}$, with $A=1.32 \times 10^{-45} \mathrm{~s}^{3}$ & \\
\hline Phonon-boundary & & $\begin{array}{l}\text { In bulk (Ref. 29): } \tau_{b}^{-1}=v_{g, s} / L F \text {, with } L=7.16 \times 10^{-3} \mathrm{~m} \text {, and } F=0.68 \\
\text { In thin films (Ref. 30): } \tau_{B}^{-1}=\frac{v_{g, s}(\vec{q})}{T_{S i}} \cdot \frac{1-p(\vec{q})}{1+p(\vec{q})} \text {, with } p(\vec{q})=\exp \left(-4 q^{2} \Delta^{2} \cos ^{2} \theta_{B}\right)\end{array}$ & \\
\hline
\end{tabular}

For acoustic modes, another generation process comes from the anharmonic decay of optical phonons into acoustic phonons, which corresponds to the term $G_{s}^{L T O \rightarrow L T A}(\vec{r}, \vec{q})$ into (3). This process gives rise to a significant energy transfer from optical to acoustic modes. This term conserves the energy of the whole phonon system. It is treated here within the approach developed in Ref. 8. In this approach, only the thirdorder processes in which the initial optical phonons decay into two lower energy acoustic modes (LA and TA) are considered.

The density $g_{2}\left(\omega, \omega_{\text {LTO }}-\omega\right)$ of final states for pairs of phonons which conserve both energy $\omega_{s_{0}}\left(\vec{q}_{0}\right)=\omega_{s_{1}}\left(\vec{q}_{1}\right)$ $+\omega_{s_{2}}\left(\vec{q}_{2}\right)$ and crystal momentum $\vec{q}_{0}=\vec{q}_{1}+\vec{q}_{2}+\vec{G}$ for an optical phonon with initial wave vector $\vec{q}_{0}$ and branch index $s_{O}$ were calculated considering a parabolic dispersion. We restrict our calculations to normal processes, i.e., with reciprocal vector $\vec{G}=0$.

The total phonon density of final states resulting from the anharmonic LTO-phonon decay was calculated by computing the decay of 3000 optical phonons uniformly distributed along the optical branches. The probability per unit of time that a LTO phonon decays into two acoustic modes of frequency $\omega$ and $\omega_{L T O}-\omega$, respectively, was obtained by restricting the sum over the branch $s$ to the values for which $\omega_{s_{0}}(\vec{q})=\omega$, as described in Ref. 34 . The density of states of LTA phonons was finally calculated by normalizing the number of LTA phonons by the number of phonons generated by optical phonon decay.

The resulting normalized distributions of LA and TA modes generated by optical phonon decay are plotted in Fig. 3. We see two peaks in this figure: one around $20 \mathrm{meV}$ which belongs to the TA branch and one around $43 \mathrm{meV}$ which belongs to the LA branch. This spectrum of final states computed via a simple analytic and isotropic dispersion, which for instance limits the TA energy at $20 \mathrm{meV}$, is in agreement with that obtained from Density-Functional Perturbation Theory calculation. ${ }^{34}$

The rate of LTO decay $G_{s}^{L T O \rightarrow L T A}(\vec{q}, \vec{r})$ is supposed to depend on the electron-LTO generation rate $G_{s}^{e-L T O}(\vec{q}, \vec{r})$ and on the probability $P_{s}^{L T O \rightarrow L T A}\left(\omega_{s}(\vec{q})\right)$ that LTO phonons decay in LTA phonons as determined previously. It writes

$$
G_{s}^{L T O \rightarrow L T A}(\vec{q}, \vec{r})=\sum_{\vec{q}_{L T O}} G^{e-L T O}\left(\vec{q}_{L T O}, \vec{r}\right) \frac{P_{s}^{L T O \rightarrow L T A}\left(\omega_{s}(\vec{q})\right)}{\left.N_{\vec{q}}\right|_{\omega_{s}}\left(\vec{q}^{\prime}\right)=\omega_{s}(\vec{q})},
$$

where $\sum G^{e-L T O}\left(\vec{q}_{L T O}, \vec{r}\right)$ is the total generation rate of LTO modes extracted from MC simulation. In stationary regime, it is exactly the number of optical phonons that decay into acoustic phonons. In (4), the probability $P_{s}^{L T O \rightarrow L T A}$ is normalized to the total number $\left.N_{\vec{q}}\right|_{\omega_{s}\left(\vec{q}^{\prime}\right)=\omega_{s}(\vec{q})}$ of phonons with wave vectors $\vec{q}^{\prime}$ satisfying the condition $\omega_{s}\left(\vec{q}^{\prime}\right)=\omega_{s}(\vec{q})$. Indeed, since an optical phonon with a given wave vector can decay into LTA modes with large spreading in wave vector, we assume that at a given frequency $\omega$, LTA phonons induced by LTO decay can have any wave vector $\vec{q}^{\prime}$ that satisfies the condition $\omega_{s}\left(\vec{q}^{\prime}\right)=\omega_{s}(\vec{q})$. This treatment of optical phonons allows us to conserve the total energy of the phonon system.

\section{Effective temperature and thermal boundary conditions}

At the nanoscale, when dealing with out-of-equilibrium phonon distributions, the concept of temperature is questionable, and even meaningless. However, the information on distribution of phonons can be conveniently reduced to an "equivalent temperature" field deduced from the local phonon energy given by the actual phonon distribution. ${ }^{29}$ In what follows, this notion of effective temperature will be useful.

As a first approximation, the source and drain contacts are assumed to be ideal phonon reservoirs at $300 \mathrm{~K}$. The thermal boundary resistance between $\mathrm{Si} / \mathrm{SiO}_{2}$ is neglected in this model, but that its presence limits heat flow into the $\mathrm{SiO}_{2}$. Our 1D model for the thermal transport also assumed

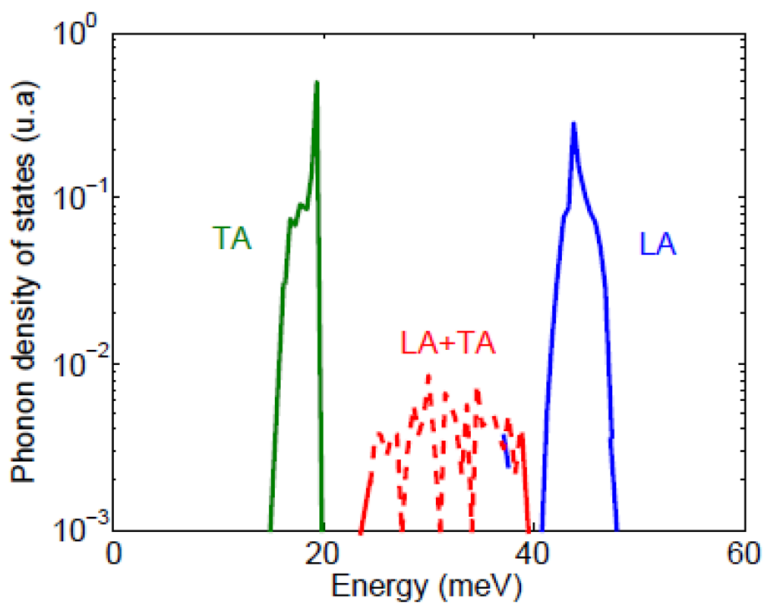

FIG. 3. Energy distribution of the phonons generated by the anharmonic decay of longitudinal optical phonons by using our calculation: LA-blue solid curve; TA green solid curve; and LA + TA red dashed curve. 
adiabatic boundary conditions (no thermal flux) along the $\mathrm{Si} / \mathrm{SiO}_{2}$ interface. Even if the $\mathrm{SiO}_{2}$ thermal conductivity is more than 1 order of magnitude lower than in confined Si, this last assumption is questionable and should be considered as a limitation of our model.

\section{PHONON GENERATION IN A DG-MOSFET}

Fig. 4(a) shows the 2D cross-section of a Si DGMOSFET that has been studied extensively in previous works by means of the eMC method. ${ }^{24}$ In this DGMOS, the silicon film thickness is fixed at $20 \mathrm{~nm}$ in order to limit the impact of quantum confinement for phonons. As we aimed at investigating the out-of-equilibrium effects, the gate length has been chosen as short as $20 \mathrm{~nm}$. This transistor should
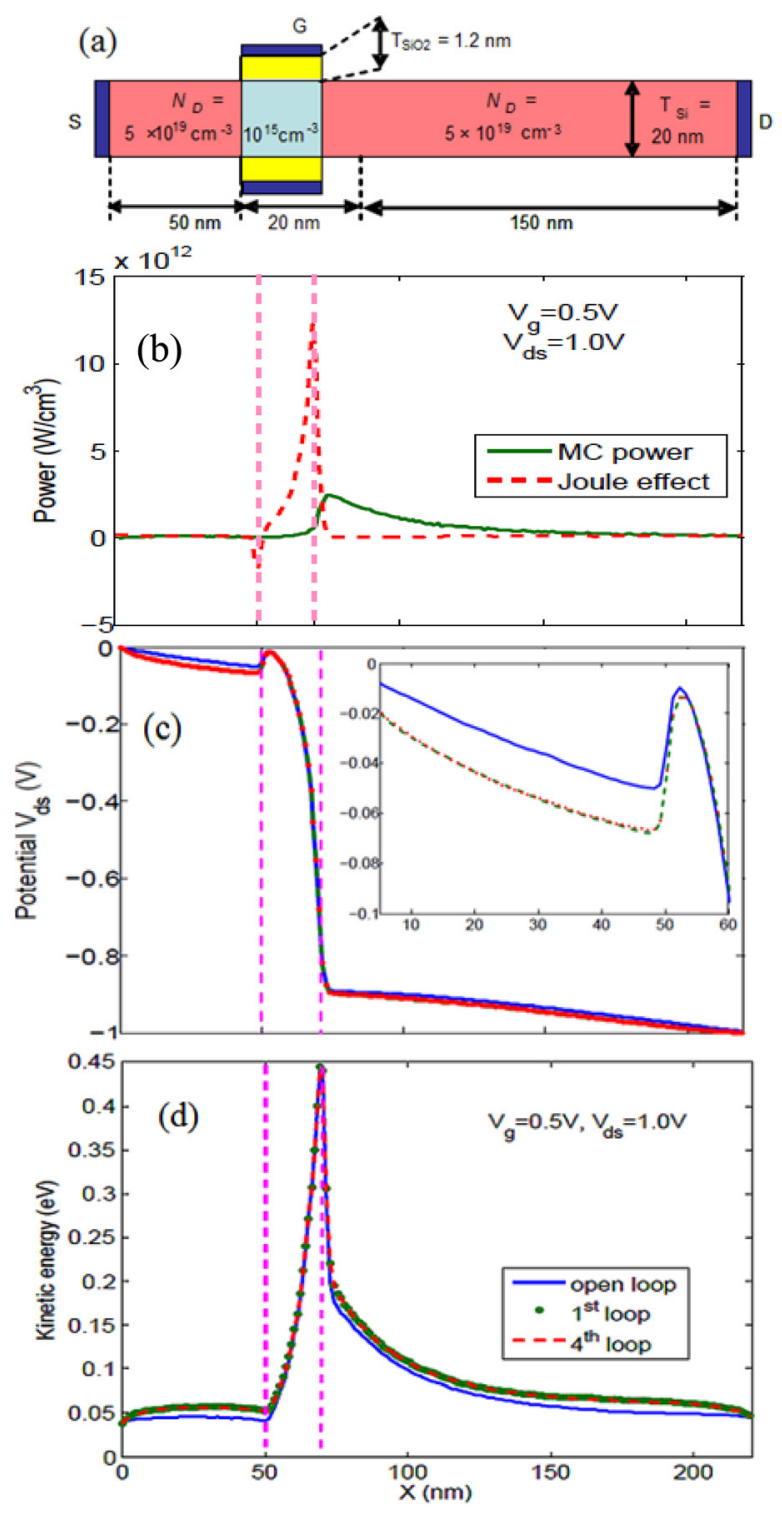

FIG. 4. (a) Schematic view of the DG-MOSFET studied in this work. (b) MC power density compared to Joule effect (green solid line) deduced from the product (dashed red line) at $V_{g s}=0.5 \mathrm{~V}, V_{d s}=1.5 \mathrm{~V}$. (c) Potential profile along the DG-MOSFET for open loop (blue curve), 1st loop (green curve) and the last (4th) loop (red curve) at $V_{g s}=0.5 \mathrm{~V}, V_{d s}=1.0 \mathrm{~V}$. Bottom: (d) profile of electron kinetic energy along the channel for $V_{g s}=0.5 \mathrm{~V}$ and $V_{d s}=1.0 \mathrm{~V}$ in the isothermal case and after self-consistent loops. exhibit poor off-state characteristics in the context of logic application, but it should be considered here as a case study chosen to illustrate the impact of strongly out-of-equilibrium transport on self-heating. The dissymmetry between source and drain region lengths will be explained afterward. The other panels of Fig. 4 show profiles of power density (Fig. 4(b)), electrostatic potential (Fig. 4(c)), and kinetic energy (Fig. 4(d)), respectively, in the channel along sourcedrain direction. They will be discussed in Subsection IV A.

In what follows, we analyze the thermal transport in this device from the solution of the pBTE by using inputs obtained from eMC simulation.

\section{A. Thermal generation}

The phonon generation in this DGMOS is first investigated through the power densities generated along the source-drain distance, plotted in Fig. 4(b) for $V_{d s}=0.5 \mathrm{~V}$ and $V_{d s}=1.5 \mathrm{~V}$. We show both the microscopic Monte Carlo result and the conventional macroscopic evaluation from the scalar product of current density $\vec{J}$ and electric field $\vec{E}$ (Local "Joule effect"). The discrepancy between these two quantities is significant. While the macroscopic calculation leads to an increasing heat generation in the channel with a maximum at the drain-end where the electric field is the highest, the MC simulation shows that the energy loss is negligible in the channel where electrons are accelerated by the field in quasi-ballistic regime. Actually, electrons lose their energy gradually in the drain extension. To quantify this decay length, fits with a simple exponential function $\left(\sim e^{-x / \lambda}\right.$ where $\lambda$ is the characteristic decay length) have been performed at $V_{g s}=0.5 \mathrm{~V}$ and for four different drain biases $V_{d s}=0.3,0.5$, 1.0 , and $1.5 \mathrm{~V}$ (Fig. 5). At $V_{d s}$ lower than $0.5 \mathrm{~V}$, the decay length is equal to $50 \mathrm{~nm}$. However, when the bias is increased, a hotspot region appears and more efficient cooling mechanisms are allowed. Thus, the parameter $\lambda$ decreases up to $28 \mathrm{~nm}$ for $V_{d s}=1.0 \mathrm{~V}$ and $22 \mathrm{~nm}$ for $V_{d s}=1.5 \mathrm{~V}$ near the hotspot while it remains close to $50 \mathrm{~nm}$ in the drain end.

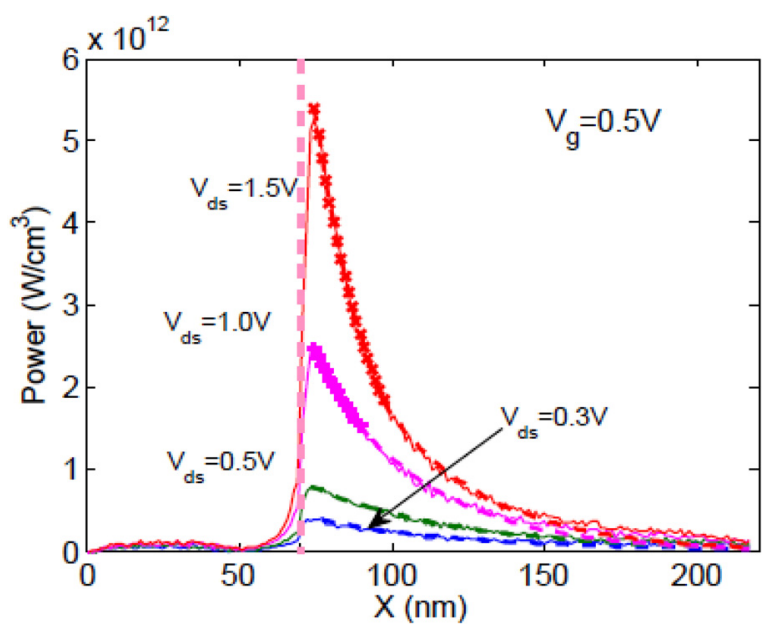

FIG. 5. MC emission power fitted in drain region in the transistor at $V_{g s}=0.5 \mathrm{~V}$ and at different $V_{d s}$ and correspondent exponential function fits. Solid lines indicate the MC powers, dashed lines are for the exponential function fits, and dashed lines with symbols are for the fit in hotspots. 
Interestingly, it appears that even in strongly nonequilibrium conditions, the total MC heating and the Joule heating are very close. Though distributed differently in space, the total electronic energy transformed into phononic energy is the same in both cases. This means that the total energy in the simulated system is actually conserved. However, the MC heat generation model is clearly essential for accurate prediction of the distribution of phonons generated in the "hot spot" inside the drain region, which will be used in Sec. $\mathrm{V}$ as an input to the phonon transport model (pBTE).

\section{B. Non-equilibrium phonon population}

The strong advantage of our code is its ability to provide the phonon distribution in both real and reciprocal spaces. Hence, it allows us to quantify the contribution of electrons to the non-equilibrium distribution of phonons. In Fig. 6, on left vertical axis, we compare the actual LA energy distribution in the hot spot resulting from the pBTE solver to the equilibrium distribution obtained from Bose-Einstein statistics at effective temperature $T_{\text {eff }}$ in the hotspot at $V_{g s}=0.5 \mathrm{~V}$ and $V_{d s}=1.2 \mathrm{~V}$. An accumulation of LA phonons is observed around $43 \mathrm{meV}$ and $50 \mathrm{meV}$.

For the sake of clarity, the difference between both distributions is plotted on the right vertical axis. We can distinguish clearly two peaks: (i) one centered on $50 \mathrm{meV}$ which corresponds to the LA phonons due to electron-phonon scattering and (ii) another one centered on $43 \mathrm{meV}$ which corresponds to LA phonons resulting from LTO phonon decay. It appears that the contribution of LTO decay to the excess density of LA phonons is more important than that of electron-LA phonon scattering. The relative excess due to these two mechanisms is about $15 \%$ (at $43 \mathrm{meV}$ ) and $8 \%$ (at

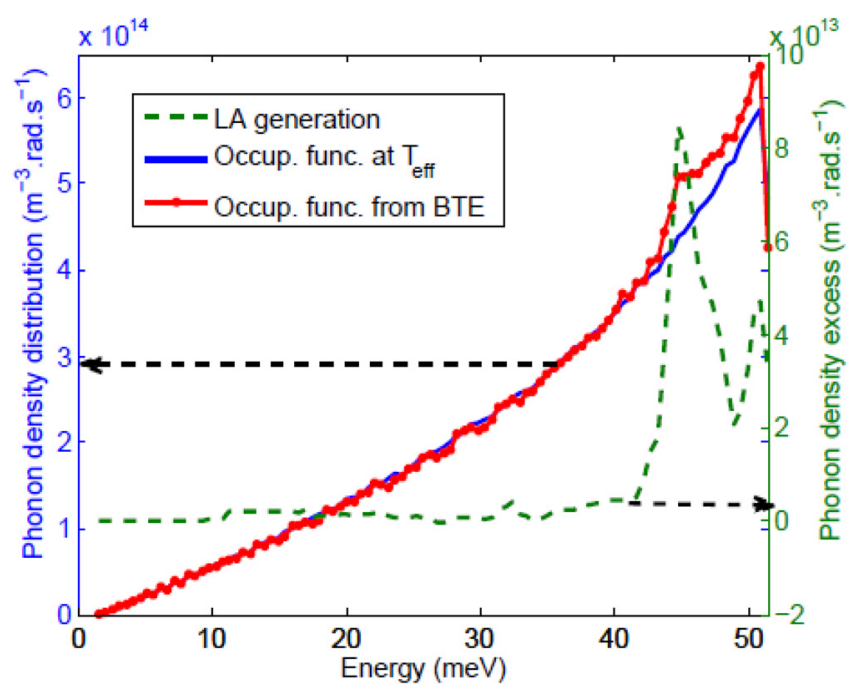

FIG. 6. LA phonon density at maximum temperature in the hot spot for $V_{g s}=0.5 \mathrm{~V}, V_{d s}=1.2 \mathrm{~V}$. Left axis: Distribution of phonon density resulting from pBTE (red line with circles) and equilibrium distribution at temperature $T_{\text {eff. }}$ Right axis: Distribution of LA phonons generated by both electronphonon scattering (around $50 \mathrm{meV}$ ) and decay of optical modes (around $43 \mathrm{meV}$ ) (dashed green line) multiplied by the relaxation time. It represents also the effective density of excess phonons compared to the equilibrium phonon density at $300 \mathrm{~K}$.
$50 \mathrm{meV}$ ), respectively, with respect to the equilibrium distribution at the effective temperature $T_{\text {eff }}$.

We also analyzed the distribution of other modes (TA, LO, and TO, not shown here), which led us to conclude that the relative difference between phonon distributions obtained from $\mathrm{pBTE}$ code and from the Bose-Einstein distribution at $T_{\text {eff }}$ is limited to a few percent. Hence, the non-equilibrium nature of the phonon transport, though visible, remains limited and the notion of phonon temperature is still acceptable in this device. The average effective temperature $T_{\text {eff }}$ appears as a good approximation of phonon mode temperature.

\section{ELECTRO-THERMAL SIMULATION IN DG-MOSFET}

\section{A. Self-consistent algorithm}

To model the effects of self-heating in transistors, the electron and phonon transport models have been selfconsistently coupled. The simulation begins with an isothermal $(300 \mathrm{~K})$ eMC simulation (called open loop). The resulting net phonon generation rates extracted as a function of position and phonon frequency are used as input for the pBTE solver that gives us the phonon temperature profiles.

As seen in Sec. IV, each mode has its own temperature and the phonon distribution of each mode can be quite well approximated by the equilibrium distribution at temperature $T=T_{\text {eff. }}$ Hence, we make this approximation for the lattice temperature. All electron scattering rates are re-calculated in each cell according to the position-dependent $T_{\text {eff }}$. Then, the eMC simulation is performed again, including the new local lattice temperature. Once the electronic system reaches the new stationary regime, the net phonon generation rates are extracted again and sent to the pBTE solver. This two-step process that successively includes one electron and one phonon transport simulation is called a loop. After each loop, the potential, the drain current, the average velocity, the average energy of electrons, and also the phonon generation rates are examined and compared with the previous loops until the convergence is reached. We have observed empirically that by using this procedure, a satisfactory convergence is reached after no more than three loops whatever the bias point.

\section{B. Temperature, potential and energy}

First, we plot in Fig. 7 at $V_{g s}=0.5 \mathrm{~V}$ and $V_{d s}=1.0 \mathrm{~V}$, after each loop, the profile of the excess effective temperature $\Delta T$ where the reference temperature is that of the contacts. The convergence of the temperature is obtained after three loops. It should be noted that the temperature in the hot spot is smaller when the self-heating is included. It reduces from $\Delta T_{\max }=140.9 \mathrm{~K}$ in the $1 \mathrm{st}$ loop to $\Delta T_{\max }=133.4 \mathrm{~K}$ when the convergence is reached.

It is interesting to examine also the effect of self-heating on the potential in the device. Fig. 4(c) displays the potential profile along the source-drain direction after different loops. The electrical resistance of the two access regions is increased in self consistent simulations due to the temperature increase. The inset shows the zoom of this potential in the source access region (mid-channel). As the temperature rises, the potential drop and the electrical resistance increase. 


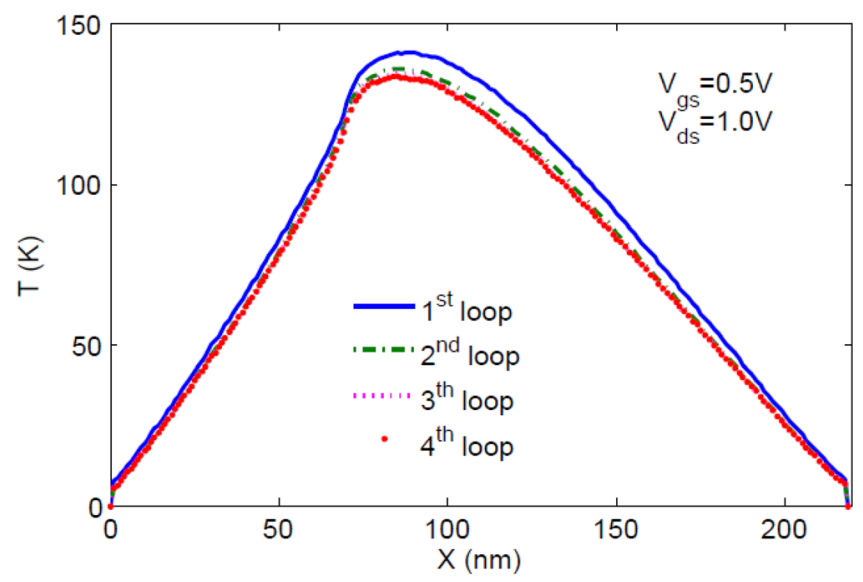

FIG. 7. Effective temperature difference $\Delta T_{\text {eff }}$ compared to the contact's temperature along the device as a function of loop number.

We can see that a good convergence is reached after the first loop.

Consistently, the thermal effects tend to enhance slightly the kinetic energy of electrons in the access regions, as a consequence of the larger potential drop. The results are shown in Fig. 4(d) for different numbers of loops. It illustrates once again the rapid convergence: after the 1st loop, the kinetic energy of electrons is very close to that obtained after the 4th loop.

It should be noted that a much greater length is needed to relax the kinetic energy in the drain extension than to relax the velocity (not shown), which is high only in the gated part of the channel. Hence, the drain access region was designed longer than the source access one, to ensure relaxation of kinetic energy relaxation before reaching the drain contact.

\section{Temperature of phonon modes}

First, we analyze the behavior of the effective temperature and of the temperature of the four phonon modes. The excess temperature profiles $(\Delta T)$ profiles in the hot spot of the DG-MOSFET under the bias condition $V_{g s}=0.5 \mathrm{~V}$, $V_{d s}=1.2 \mathrm{~V}$ are displayed in Fig. 8 .

Actually, in this out-of-equilibrium regime, the phononmodes and effective temperatures are different in the hot spot region, just beyond the gated part of the channel, where many phonons are generated by hot electrons. The LTO temperatures are slightly higher than the Fourier temperature. However, the TA phonon temperature is significantly higher because these phonons are generated by two processes, i.e., by electron-phonon scattering and by optical phonon decay, the latter mechanism being an important source of LTA phonons. Note that with our set of deformation potentials, the power density of generated LTO phonons reaches up to about $50 \%$ of the total power density (see Fig. 2). With the hypothesis that at steady-state, all LTO phonons decay into LA and TA phonons, the density of LA and TA phonons produced by this process is comparable to the density of LTO phonons. In addition, the number of LTA phonons generated by LTO phonon decay is equally distributed between LA modes and TA modes. However, at a given temperature, the equilibrium phonon occupation number of TA modes is

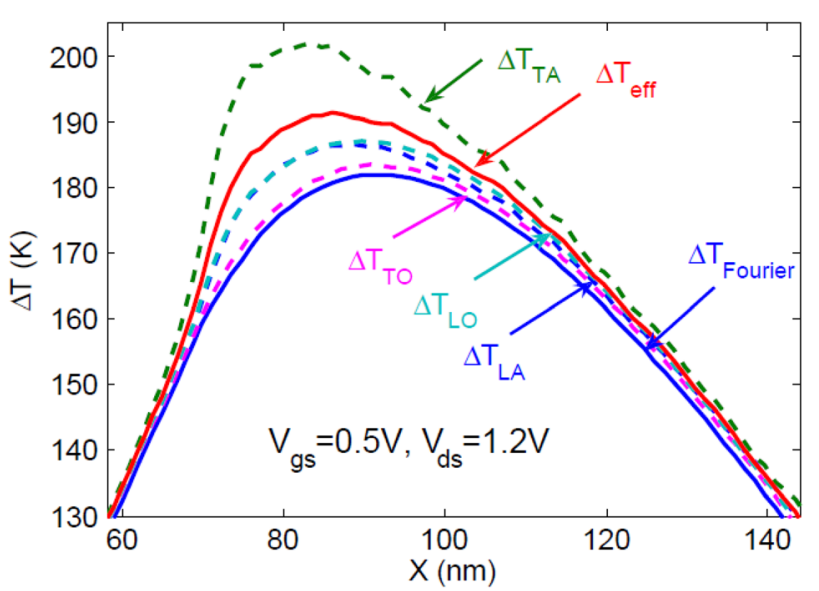

FIG. 8. Temperature difference profiles $\Delta T$ compared to the contact's temperature in the hot spot region under the bias condition $V_{g s}=0.5 \mathrm{~V}$, $V_{d s}=1.2 \mathrm{~V}: \Delta T_{L A}$ (blue dashed line), $\Delta T_{T A}$ (green dashed line), $\Delta T_{L O}$ (azure dashed line), $\Delta T_{T O}$ (purple dashed line), $\Delta T_{\text {Fourier }}$ (blue solid line), and $\Delta T_{\text {eff }}$ (red solid line).

smaller than that of LA modes. Hence, the impact of LTO decay is stronger for TA phonons than for LA phonons, which manifests through the TA temperature that is much higher than the LA temperature. This is the origin of the strong enhancement of the TA temperature in the hot spot region. The diffusive Fourier model underestimates the temperature $\left(\Delta T_{\text {Fourier_max }}=182.0 \mathrm{~K}\right.$ at $\left.X=91 \mathrm{~nm}\right)$ in the hotspot region compared to the average effective temperature obtained from the BTE model $\left(\Delta T_{\text {eff } \max }=191.3 \mathrm{~K}\right.$ at $X=86 \mathrm{~nm})$.

Comparison between the excess effective temperature $\Delta T$ given by pBTE simulation and the excess Fourier temperature for $V_{g s}=0.5 \mathrm{~V}$ and for different drain voltages $V_{d s}=0.5,1.0$, and $1.2 \mathrm{~V}$ are shown in Fig. 9. The effect of self-consistent simulation is more important under high drain bias. Discontinuities of temperature are also visible near the isothermal boundaries, which is consistent with previous results in Si bars. ${ }^{35}$ The shorter the device is, the stronger the discontinuities at the boundaries are. This effect is a consequence of out-of-equilibrium phonon transport which may survive even near the equilibrium contacts (see also the

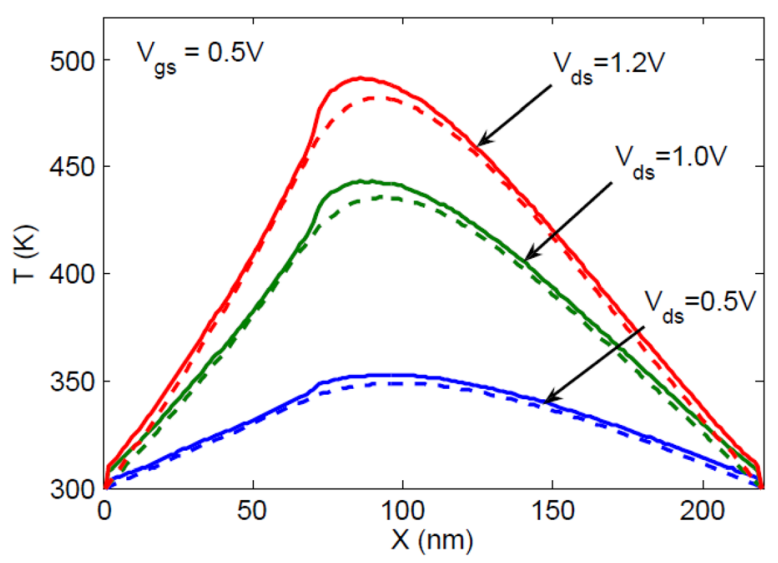

FIG. 9. Drain bias dependence of excess temperature profile with respect to the contact temperature and $V_{g s}=0.5 \mathrm{~V}$. Excess temperatures $\Delta T_{F o u r i e r}$ and $\Delta T_{\text {eff }}$ are plotted in dashed and solid lines, respectively. 
discussion in Ref. 29). This effect has been observed in $\mathrm{Si}$ bars of length up to $5 \mu \mathrm{m}$. ${ }^{35}$

\section{Electro-thermal simulation: Heat transport and consequences}

To identify the most important scattering mechanisms and the influence of self-heating on the scattering processes, the number of electron interactions with impurities, intravalley acoustic phonons, intervalley phonons, and rough interfaces has been collected during 10000 time steps of eMC simulation in two cases: isothermal and electro-thermal case. In Fig. 10, we plot these interaction numbers along the gated part of the channel for $V_{g s}=0.5 \mathrm{~V}$ and $V_{d s}=1.0 \mathrm{~V}$. The intervalley interaction is the scattering mechanism the most sensitive to self-heating. In particular, at the channel drain-end, the number of intervalley scattering events in coupled simulation is enhanced by $45.6 \%$ with respect to the case of isothermal simulation.

The increased numbers of scattering events along the device reduce the fraction of ballistic electrons that is quantified by the intrinsic ballisticity $B_{\text {int }}$. This quantity $B_{\text {int }}$ corresponds to the percentage of purely ballistic electrons at the drain-end of the gated part of channel. The definition and the counting procedure in eMC simulation have been detailed in Ref. 23. In Fig. 11, we plot the evolution of the percentage of ballistic electrons along the channel for $V_{g s}=0.5 \mathrm{~V}$ and for two drain biases, $V_{d s}=0.5 \mathrm{~V}$ and $V_{d s}=1.0 \mathrm{~V}$. The higher the drain bias, the smaller is the ballisticity. At the drain-end of the channel, for $V_{d s}=1.0 \mathrm{~V} B_{\text {int }}$ falls from $43 \%$ in the isothermal case to $35 \%$ in the electro-thermal simulation.

The evolution of the $I_{d}-V_{d s}$ characteristics at $V_{g s}=0.5 \mathrm{~V}$ is shown in Fig. 12. Once again, it is observed that the convergence is reached after only one or two eMC/pBTE steps. A relative current reduction of $6.9 \%$ and $8.1 \%$ of the drain current is observed for $V_{d s}=0.5 \mathrm{~V}$ and $1.0 \mathrm{~V}$, respectively, for a roughness of $3 \mathrm{~nm}$. This degradation of current can be understood as a direct consequence of the increase of

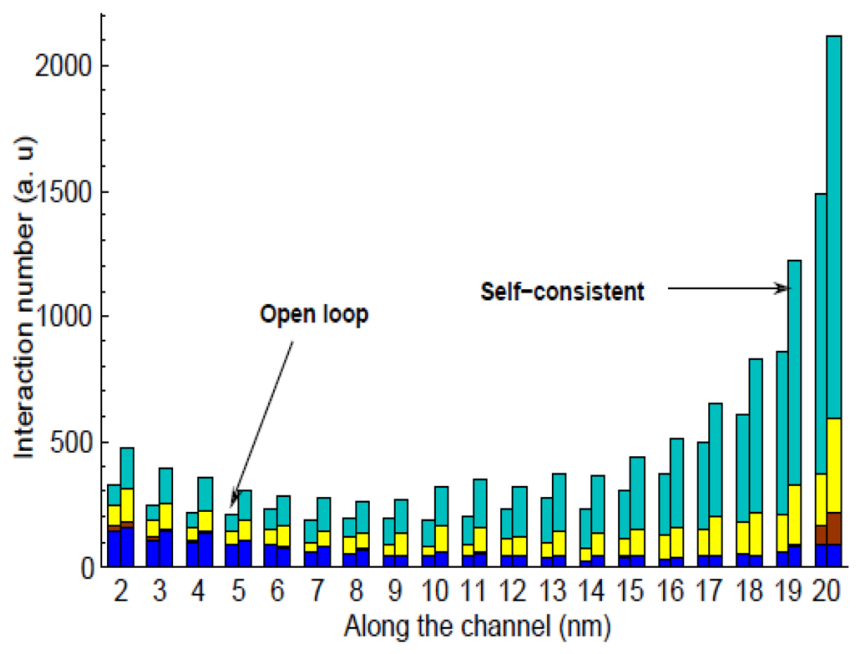

FIG. 10. Distribution of interaction number along the channel as a function of interaction types in the DG-MOSFET at $V_{g s}=0.5 \mathrm{~V}, V_{d s}=1.0 \mathrm{~V}$ in both isothermal and electro-thermal case.

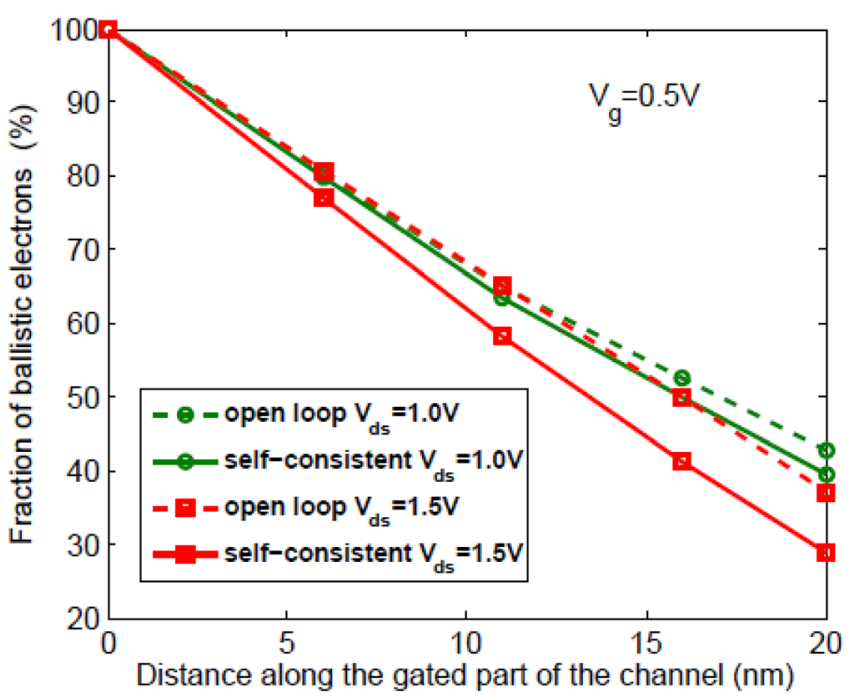

FIG. 11. Evolution of the fraction of ballistic electrons along the channel in the DG-MOSFET at $V_{g s}=0.5 \mathrm{~V}, V_{d s}=0.5 \mathrm{~V}$, and $1.0 \mathrm{~V}$.

scattering event number in the active region of the device due to self-heating effects.

\section{CONCLUSION}

In this work, a new self-consistent electron-phonon transport model is presented. The phonon transport model includes both the phonon generation rates given by the electron Monte Carlo simulation and the decay of optical phonons into acoustic modes. The profiles of temperature per mode and the comparison between diffusion temperature and the effective temperature are analyzed. Our results show the significant role of LTO decay in heat transport, particularly in the hotspot region.

The BTE solver provides very detailed insight into electro-thermal effects at the nanoscale, which have been analyzed in a $20 \mathrm{~nm}$-long DG-MOSFETs in terms of phonon distribution, effective temperature, potential, electron energy, and terminal current. The electro-thermal effects have been shown to increase the scattering rates of electrons

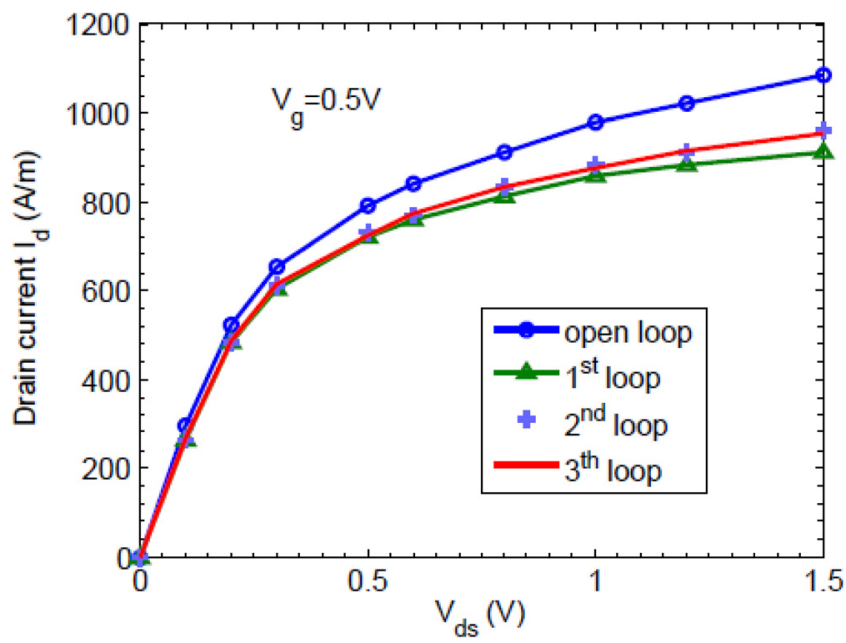

FIG. 12. $I_{d}-V_{d s}$ curves for $V_{g s}=0.5 \mathrm{~V}$ after open loop, the $1 \mathrm{st}$ loop, the 2 nd loop and the last loop (4th loop). 
along the device, and thus to reduce the intrinsic ballisticity, which is at the origin of the self-heating-induced degradation of current.

This study has shown that self-heating effects have limited impact on the DC characteristics in this particular device, in spite of strong out-of-equilibrium electron and phonon behavior. It could be extended in future works to investigate their influence on high-frequency performance and noise characteristics, and to consider other designs with thinner channel.

\section{ACKNOWLEDGMENTS}

This work was partially supported by the French ANR through project NOE (No. 12JS03-006-01).

${ }^{1}$ O. Muscato, V. Di Stefano, and C. Milazzo, J. Comput. Electron. 7, 142 (2008).

${ }^{2}$ K. Etessam-Yazdani, R. Hussin, and M. Asheghi, in Proceedings of InterPACK 2005, San Francisco, USA, July 17-22 (2005), pp. 2087-2095.

${ }^{3}$ J. H. Chun, B. Kim, Y. Liu, O. Tornblad, and R. W. Dutton, in Proceedings of International Conference on Simulation of Semiconductor Processes and Devices (SISPAD 2005), Tokyo, Japan, September 3-5 (2005), pp. 275-278.

${ }^{4}$ O. Muscato and V. Di Stefano, COMPEL: Int. J. Comput. Math. Electr. Electron Eng. 30, 519-537 (2011).

${ }^{5}$ E. Pop, R. W. Dutton, and K. E. Goodson, J. Appl. Phys. 96, 4998 (2004).

${ }^{6}$ E. Pop, R. W. Dutton, and K. E. Goodson, Appl. Phys. Lett. 86, 082101 (2005).

${ }^{7}$ S. Sinha, E. Pop, R. W. Dutton, and K. E. Goodson, J. Heat Transfer 128, 638 (2005).

${ }^{8}$ J. Rowlette and K. E. Goodson, IEEE Trans. Electron Devices 55, 220 (2008).

${ }^{9}$ E. Pop, Nano Res. 3, 147 (2010).

${ }^{10}$ M. Zebarjadi, A. Shakouri, and K. Esfarjani, Phys. Rev. B 74, 195331 (2006).

${ }^{11}$ T. Sadi and R. W. Kelsall, J. Appl. Phys. 107, 064506 (2010).
${ }^{12}$ T. Sadi and R. W. Kelsall, J. Comput. Electron. 11, 118 (2012).

${ }^{13}$ J. Lai and A. Majumdar, J. Appl. Phys. 79, 7353 (1996).

${ }^{14}$ K. Raleva, D. Vasileska, and S. M. Goodnick, IEEE. Trans. Electron Devices 55(6), 1306 (2008).

${ }^{15}$ D. Vasileska, K. Raleva, and S. M. Goodnick, IEEE Trans. Electron Devices 56(12), 3064 (2009).

${ }^{16}$ D. Vasileska, K. Raleva, A. Hossain, and S. M. Goodnick, J. Comput. Electron. 11, 238 (2012).

${ }^{17}$ T. Hatakeyamaa and K. Fushinobua, Heat Transfer Eng. 29, 120 (2008).

${ }^{18}$ C. Ni, Z. Aksamija, J. Y. Murthy, and U. Ravaioli, J. Compt. Electron. 11, 93 (2012).

${ }^{19}$ Y. Kamakura, N. Mori, K. Taniguchi, T. Zushi, and T. Watanabe, in Proceedings of International Conference on Simulation of Semiconductor Processes and Devices (SISPAD 2010), Bologna, Italy, September 6-8 (2005), pp. 89-92.

${ }^{20}$ C. Ni, Ph.D. dissertation, Purdue University, 2009.

${ }^{21}$ A. Bournel, P. Dollfus, E. Cassan, and P. Hesto, Appl. Phys. Lett. 77, 2346 (2000).

${ }^{22}$ J. Saint-Martin, A. Bournel, and P. Dollfus, IEEE Trans. Electron Devices 51, 1148 (2004).

${ }^{23}$ P. Dollfus, A. Bournel, S. Galdin-Retailleau, S. Barraud, and P. Hesto, IEEE Trans. Electron Devices 51, 749 (2004).

${ }^{24}$ J. Saint-Martin, A. Bournel, F. Monsef, C. Chassat, and P. Dollfus, Semicond. Sci. Technol. 21, L29 (2006).

${ }^{25}$ V. Aubry-Fortuna, P. Dollfus, and S. Galdin-Retailleau, Solid-State Electron. 49, 1320 (2005).

${ }^{26}$ P. Dollfus, J. Appl. Phys. 82, 3911-3916 (1997).

${ }^{27}$ J. Sjakste, N. Vast, H. Jani, S. Obukhov, and V. Tyuterev, Phys. Status Solidi B 250, 716-720 (2013).

${ }^{28}$ P. G. Klemens, Proc. R. Soc. London, Ser. A 208, 108 (1951).

${ }^{29}$ T. T. Trang Nghiêm, Ph.D. dissertation, University of Paris-Sud, Orsay, France, 2013.

${ }^{30}$ M. G. Holland, Phys. Rev. 132, 2461 (1963).

${ }^{31}$ R. Berman, F. E. Simon, and J. M. Ziman, Proc. R. Soc. London, Ser. A 220, 171 (1953).

${ }^{32}$ G. Lang, K. Karch, M. Schmitt, P. Pavone, A. P. Mayer, R. K. Wehner, and D. Strauch, Phys. Rev. B 59, 6182 (1999).

${ }^{33}$ W. Liu and M. Asheghi, J. Appl. Phys. 98, 123523 (2005).

${ }^{34}$ A. Debernardi, S. Baroni, and E. Molinari, Phys. Rev. Lett. 75, 1819 (1995).

${ }^{35}$ B. Wong, M. Francoeur, and M. P. Mengüç, Int. J. Heat Mass Transfer 54, 1825 (2011). 